\title{
High-power broadband supercontinuum generation through a simple narrow-bandwidth FBGs-based fiber laser cavity
}

\author{
Song Zhang (张 嵩), Man Jiang (姜 曼), Can Li (李 灿), Rongtao Su (粟荣涛), Pu Zhou (周 朴), and \\ Zongfu Jiang (姜宗福) \\ College of Advanced Interdisciplinary Studies, National University of Defense Technology, Changsha 410073, China \\ *Corresponding author: jiangman7@126.com \\ Received October 9, 2021 | Accepted November 19, 2021 | Posted Online December 14, 2021
}

\begin{abstract}
The generation of supercontinuum [SC often requires ultrashort pulsed lasers with high peak power and gain media with large nonlinear coefficients, such as a long piece of fiber or photonic crystal fiber. In this Letter, we propose and demonstrate that high-power SC can be generated through a simple narrow-bandwidth fiber Bragg gratings (FBGs)-based laser cavity without any modulation, based on the mechanism of intense nonlinear effects induced by the inherent self-pulsation generated inside the cavity. In the experiment, an $\sim 80 \mathrm{~W}$ SC laser with the spectrum range from $<600 \mathrm{~nm}$ to $1600 \mathrm{~nm}$ was achieved. To the best of our knowledge, this is the first report about SC generation through a simple fiber laser cavity. This work enriches the research content of SC and provides a cost-effective method for high-power SC lasers.
\end{abstract}

Keywords: supercontinuum; fiber laser; narrow-linewidth laser.

DOI: 10.3788/COL202220.011405

\section{Introduction}

The supercontinuum (SC) has both the wide spectral characteristics of traditional broadband light and the high spatial coherence of lasers. Because of this advantage, the SC has a wide range of applications, such as spectroscopy, biomedicine, and fiber optic communications ${ }^{[1-4]}$, and high-power operation is required to increase the system performance and capability ${ }^{[5-7]}$.

Currently, the mainly used ways to generate high-power SC can be categorized two types. The first way is to use high-power continuous wave or pulsed fiber laser to pump photonic crystal fiber $(\mathrm{PCF})^{[8-14]}$. With the help of a high nonlinear coefficient of PCF, the pump laser spectrum will be greatly broadened in it and become an SC. Recently in 2021, researchers from the China Academy of Engineering Physics achieved $>300 \mathrm{~W}$ SC laser based on the "pulsed fiber laser + PCF" method ${ }^{[8]}$. The output spectrum extended from 390 to $2400 \mathrm{~nm}$ with good flatness. The other way is to directly generate SC in the nonlinear fiber amplifier ${ }^{[15-18]}$. It usually employs a high peak-power pulsed laser as the seed laser for injection into the fiber amplifier, in which the laser peak power can be boosted and induce the strong nonlinear effects to realize SC output. In 2013, Song et al. ${ }^{[18]}$ realized a 177 W SC laser based on "nanosecond fiber laser + three-stage nonlinear fiber amplifiers." The spectrum covered 1064$2000 \mathrm{~nm}$ with a $-10 \mathrm{~dB}$ spectral width of $740 \mathrm{~nm}$. In addition, a new method of generating SC has emerged, which is about using random fiber lasers (RFLs) to directly generate near-infrared $S^{[19-22]}$. Researchers from the University of Electronic Science and Technology of China firstly, to the best of our knowledge, reported this method in $2018^{[19]}$, and they used a $1365 \mathrm{~nm}$ Raman fiber laser to pump an RFL with a working wavelength of $1461 \mathrm{~nm}$. The collective effects of RFL, stimulated Raman scattering (SRS) and cross-phase modulation (XPM) finally lead to spectral broadening, and the SC was generated with $-20 \mathrm{~dB}$ bandwidth of $145 \mathrm{~nm}$.

Here, we demonstrated a novel scheme to generate highpower SC. Different from previous methods, this scheme is based on a simple fiber Bragg gratings (FBGs)-based laser cavity. When the bandwidth of the FBGs pair is narrow, random selfpulses will be generated with the peak power much higher than the average power ${ }^{[23-27]}$, which will cause nonlinear effects and broaden the laser spectrum. In the experiment, we built a narrow-linewidth laser cavity with a pair of polarizationmaintaining (PM) FBGs. Applying temperature control to FBGs, the SC with an average power of $\sim 80 \mathrm{~W}$ was achieved, and the optical-to-optical conversion efficiency was $43.2 \%$. The output spectrum ranged from $<600 \mathrm{~nm}$ to $1600 \mathrm{~nm}$, and the $-10 \mathrm{~dB}$ bandwidth was up to $420.3 \mathrm{~nm}$ with $1250 \mathrm{~nm}$ as the central wavelength. 


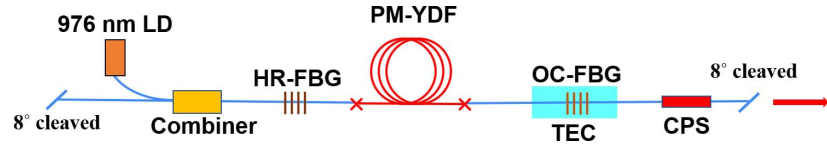

Fig. 1. Structural diagram of fiber laser cavity.

\section{Experimental Setup}

The structure of the fiber laser cavity is shown in Fig. 1. This cavity was constructed through cross splicing a pair of PM FBGs, in which the reflectivity of the highly-reflected FBG (HR-FBG) and output-coupling FBG (OC-FBG) was $99.5 \%$ and $10 \%$, respectively. Cross-splicing techniques made the laser with a linear polarization state parallel to the fast axis of $\mathrm{HR}-\mathrm{FBG}^{[28]}$. The $-3 \mathrm{~dB}$ bandwidths were $0.28 \mathrm{~nm}$ and $0.08 \mathrm{~nm}$, corresponding to the HR-FBG and OC-FBG. The laser gain was provided by a piece of $4.5 \mathrm{~m}$ PM ytterbium-doped fiber (YDF) with a core-clad diameter of $10 / 125 \mu \mathrm{m}$ and a core numerical aperture of 0.07 . The cladding absorption coefficient of YDF was $4.95 \mathrm{~dB} / \mathrm{m}$ at $976 \mathrm{~nm}$. The cavity was directly pumped by a $976 \mathrm{~nm}$ laser diode (LD) through a combiner, and the pump power could reach $\sim 185 \mathrm{~W}$. The unabsorbed pump power was removed by a cladding power stripper (CPS). While the temperature of HR-FBG was maintained at the room temperature of $21^{\circ} \mathrm{C}$, the OC-FBG was set to $12^{\circ} \mathrm{C}$ by a thermal electrical cooler (TEC). It was found in the experiment that such a temperature difference can ensure that the laser can resonate normally ${ }^{[29]}$ and play a key role in the generation of SC. Both ends of the fiber laser cavity were cleaved with $8^{\circ}$ to prevent parasitic oscillation.

\section{Results and Discussion}

When the injected pump power was $27.3 \mathrm{~W}$, the laser power reached the power of $13.8 \mathrm{~W}$. At this power, the mean normalized laser timing sequences and spectrum were measured and are shown in Figs. 2(a) and 2(b). All of the laser timing sequences in this Letter were gathered by a high-speed photodetector with $5 \mathrm{GHz}$ bandwidth and an oscilloscope with $5 \mathrm{GSa} / \mathrm{s}$. The sampling time interval was $0.2 \mathrm{~ns}$, and the measurement time window was $40 \mu \mathrm{s}$. It should be noted that the $y$ axes' units of laser timing sequences in Fig. 2 are the relative voltage value, and the relationship between power and voltage can be descripted as $P \propto U^{2}$. The random self-pulses were observed at the power of $13.8 \mathrm{~W}$, in which the peak-power values were 9-25 times the average power. At this time, the output spectrum had narrow linewidth, and the $-3 \mathrm{~dB}$ bandwidth was $58 \mathrm{pm}$.

With the further increase of pump power, the high peak-value pulse occurred at the laser power of $44.1 \mathrm{~W}$, as shown in Fig. 2(c). The peak power can reach as high as the kilowatt $(\mathrm{kW})$ level. Within $40 \mu$ s, there were statistically $\sim 35$ pulses with peak power of $>100$ times the average power. These high-power pulses induced the nonlinear effects and led to spectral broadening. The Raman Stokes light was observed in the spectral diagram, as shown in the Fig. 2(d), and there had been some signs in the spectrum about the generation of SC.

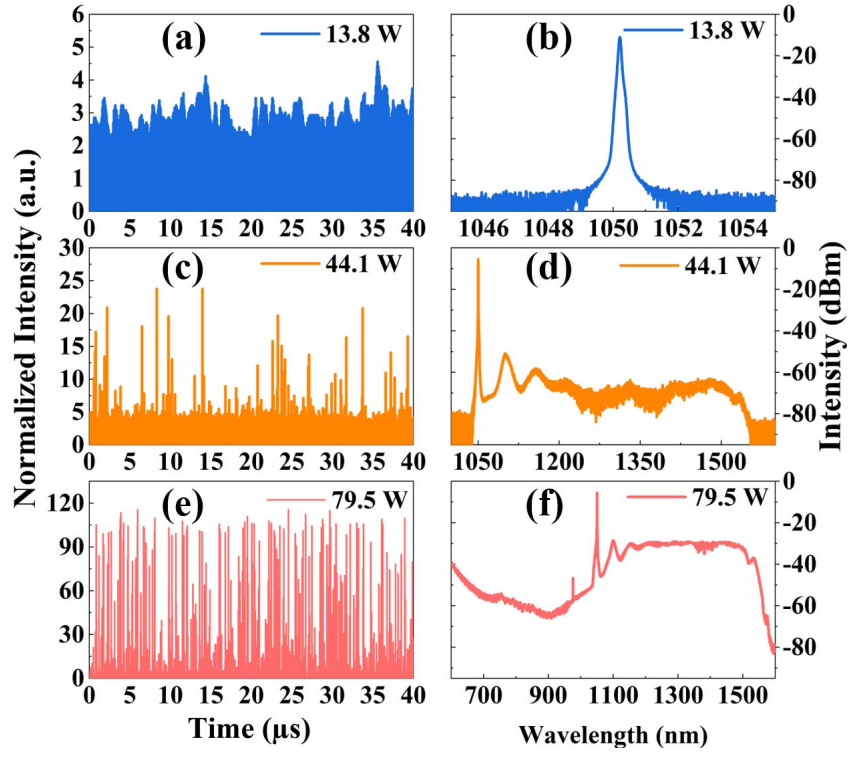

Fig. 2. Laser timing sequences and spectra at different powers.

When the pump power was increased to $183.9 \mathrm{~W}$, the laser power reached $79.5 \mathrm{~W}$ with the optical-to-optical conversion efficiency of $43.2 \%$. The peak power of the random pulse had astonishingly reached $>10^{4}$ times the average power, which means that the instantaneous laser power had reached as high as the $0.8 \mathrm{MW}$ level, as shown in Fig. 2(e). High peak-value pulses appeared more frequently, and $\sim 1779$ pulses with peak power of $>2.5 \times 10^{3}$ times the average power occurred within $40 \mu \mathrm{s}$. These pulses' duration presented random characteristics and fluctuated within the range of 1-10 ns. Such intense and frequent pulses caused more severe nonlinear effects, and the SC was generated, as shown in Fig. 2(f). The spectral range was from $<600 \mathrm{~nm}$ to $1600 \mathrm{~nm}$. The detection of shorter wavelengths was limited by the wavelength range of the optical spectrum analyzer $(600-1700 \mathrm{~nm})$. The $-10 \mathrm{~dB}$ bandwidth was $420.3 \mathrm{~nm}$ with $1250 \mathrm{~nm}$ as the central wavelength. The SRS was the most obvious nonlinear phenomenon in our experiment, and its classic formula of the power threshold is depicted as the following ${ }^{[30]}$ :

$$
P_{\text {th }}=16 A_{\text {eff }} / g_{R} L_{\text {eff }} \text {, }
$$

where $P_{\text {th }}$ is the SRS threshold, $A_{\text {eff }}$ is the effective area of the fiber laser, $g_{R}=10^{-13} \mathrm{~m} / \mathrm{W}$ is the peak value of the SRS gain spectrum, and $L_{\text {eff }}$ is the effective nonlinear interaction length; the SRS threshold calculated in our fiber cavity should be at the $2 \mathrm{~kW}$ level. In other words, it is impossible to observe Raman Stokes light on the spectrum with laser power of $80 \mathrm{~W}$. However, the SRS response time is in the order of hundreds of femtoseconds ${ }^{[30]}$, and the nanosecond (ns)-scale self-pulses peak power had reached the 0.8 MW level. So, the Raman Stokes light observed in the spectrum was attributed to these strong self-pulses. The physical mechanism behind these random strong self-pulses is intriguing, and we speculated that they 


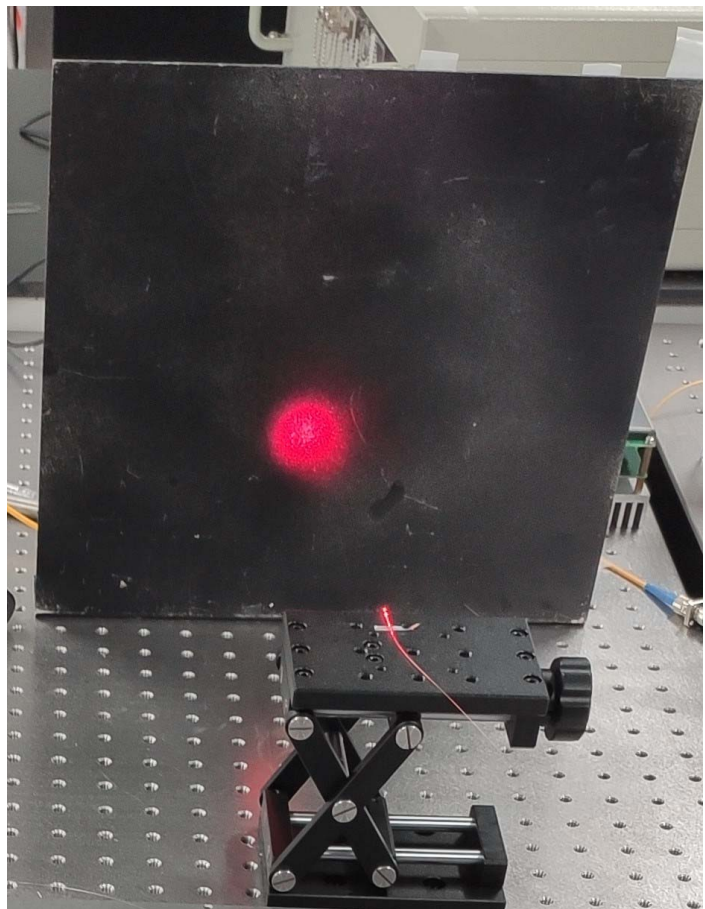

Fig. 3. Visible red laser light at the power of $79.5 \mathrm{~W}$.

are especially related to the stimulated Brillouin scattering $(\mathrm{SBS})^{[31-33]}$.

Since the wavelength of SC had been extended to the visible light band, the visible red laser output was observed in the experiment and photographed, as shown in Fig. 3. In addition, it can be seen from Fig. 4 that the laser power increased linearly with pump power, indicating that this kind of SC laser has potential for further power boosting.

For testing the stability of SC generated through this simple narrow-bandwidth FBGs-based laser cavity, the measured spectra of $\sim 70 \mathrm{~W}$ SC laser at the different dates (May 31, 2021 and Sep 15, 2021) are shown in Fig. 5. It turns out that the two output spectra agree very well before the wavelength of $1420 \mathrm{~nm}$, and

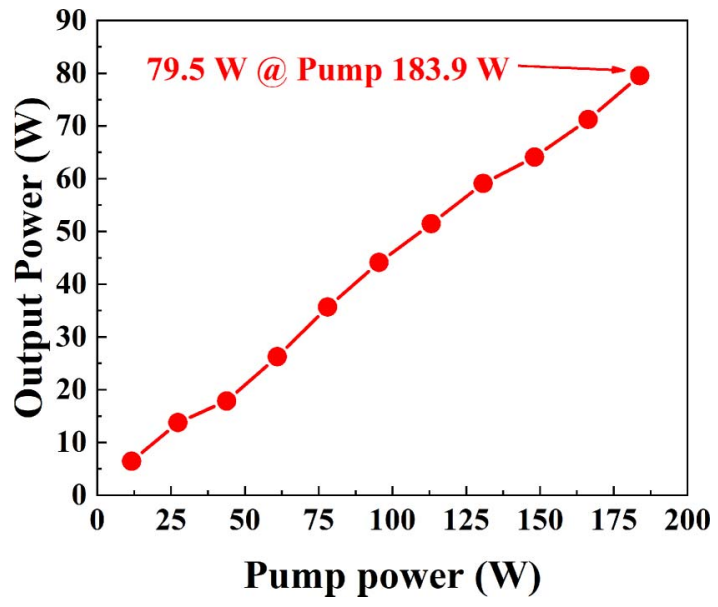

Fig. 4. Laser power versus pump power.

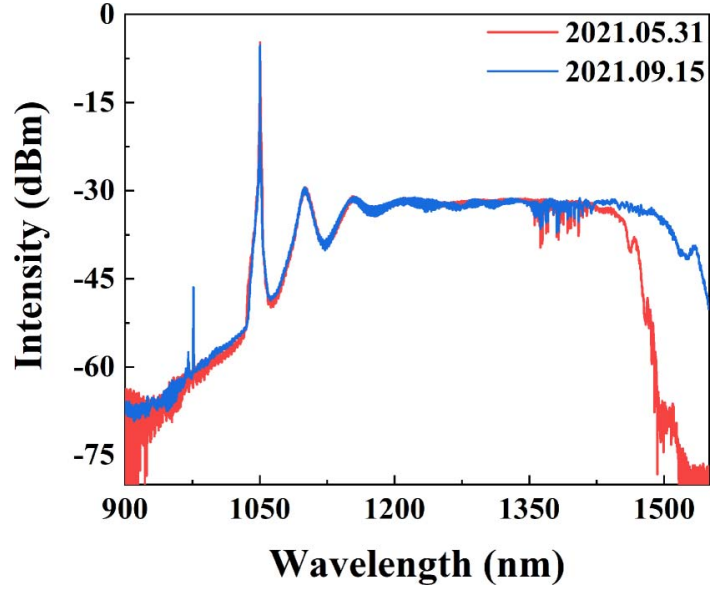

Fig. 5. Spectra of SC at the different dates.

the difference is only reflected in the longer wave band, which was attributed to the random characteristic of self-pulses or optical rogue waves ${ }^{[23,26,27,31-33]}$.

\section{Conclusions}

In conclusion, we demonstrated a novel way to generate highpower SC by employing the self-pulsing effect in a narrow-linewidth fiber laser cavity. With the help of temperature control of FBGs, the $\sim 80$ W SC was finally achieved. The output spectrum ranged from $<600 \mathrm{~nm}$ to $1600 \mathrm{~nm}$, and the $-10 \mathrm{~dB}$ bandwidth was $420.3 \mathrm{~nm}$ with $1250 \mathrm{~nm}$ as the central wavelength. In the future, we will work on a theoretical model about the production mechanism of those high peak-power random pulses and further boost the SC output power through bidirectional pumping.

\section{Acknowledgement}

This work was supported by the National Natural Science Foundation of China (Nos. 62035015 and 62005316) and the Innovative Research Groups of Hunan Province (No. 2019JJ10005).

\section{References}

1. J. C. Travers, "Blue extension of optical fibre supercontinuum generation," J. Opt. 12, 113001 (2010).

2. C. R. Petersen, N. Prtljaga, M. Farries, J. Ward, B. Napier, G. R. Lloyd, J. Nallala, N. Stone, and O. Bang, "Mid-infrared multispectral tissue imaging using a chalcogenide fiber supercontinuum source," Opt. Lett. 43, 999 (2018).

3. J. Park, D. J. Brady, G. Zheng, L. Tian, and L. Gao, "Review of bio-optical imaging systems with a high space-bandwidth product," Adv. Photon. 3, 044001 (2021)

4. N. M. Israelsen, C. R. Petersen, A. Barh, D. Jain, M. Jensen, G. Hannesschlager, P. Tidemand-Lichtenberg, C. Pedersen, A. Podoleanu, and O. Bang, "Real-time high-resolution mid-infrared optical coherence tomography," Light Sci. Appl. 8, 11 (2019). 
5. T. Hakala, J. Suomalainen, S. Kaasalainen, and Y. Chen, "Full waveform hyperspectral LiDAR for terrestrial laser scanning," Opt. Express 20, 7119 (2012).

6. C. J. Zarobila, J. J. Butler, H. J. Patrick, X. Xiong, and X. Gu, "Supercontinuum fiber laser source for reflectance calibrations in remote sensing," Proc. SPIE 7807, 78070B (2010)

7. V. V. Alexander, Z. Shi, M. N. Islam, K. Ke, G. Kalinchenko, M. J. Freeman, A. Ifarraguerri, J. Meola, A. Absi, J. Leonard, J. A. Zadnik, A. S. Szalkowski, and G. J. Boer, "Field trial of active remote sensing using a high-power shortwave infrared supercontinuum laser," Appl. Opt. 52, 6813 (2013).

8. H. Zhang, F. Li, R. Liao, K. Dong, Y. Li, H. Lin, J. Wang, and F. Jing, "Supercontinuum generation of $314.7 \mathrm{~W}$ ranging from 390 to $2400 \mathrm{~nm}$ by tapered photonic crystal fiber," Opt. Lett. 46, 1429 (2021).

9. Z. Li, Z. Jia, C. Yao, Z. Zhao, N. Li, M. Hu, Y. Ohishi, W. Qin, and G. Qin, "22.7 W mid-infrared supercontinuum generation in fluorotellurite fibers," Opt. Lett. 45, 1882 (2020).

10. X. Wang, C. Yao, P. Li, Y. Wu, L. Yang, G. Ren, and C. Wang, "All-fiber highpower supercontinuum laser source over 3.5 microm based on a germaniacore fiber," Opt. Lett. 46, 3103 (2021).

11. J. C. Travers, A. B. Rulkov, B. A. Cumberland, S. V. Popov, and J. R. Taylor, "Visible supercontinuum generation in photonic crystal fibers with a $400 \mathrm{~W}$ continuous wave fiber laser," Opt. Express 16, 14435 (2008).

12. Y. Zhu, Z. Zheng, X. Ge, G. Du, S. Ruan, C. Guo, P. Yan, P. Hua, L. Xia, and Q. Lü, "High-power, ultra-broadband supercontinuum source based upon 1/ $1.5 \mu \mathrm{m}$ dual-band pumping," Chin. Opt. Lett. 19, 041403 (2021).

13. Y. Tao and S.-P. Chen, "All-fiber high-power linearly polarized supercontinuum generation from polarization-maintaining photonic crystal fibers," High Power Laser Sci. Eng. 7, e28 (2019).

14. C. Huang, M. Liao, W. Bi, X. Li, L. Hu, L. Zhang, L. Wang, G. Qin, T. Xue, D. Chen, and W. Gao, "Ultraflat, broadband, and highly coherent supercontinuum generation in all-solid microstructured optical fibers with all-normal dispersion," Photon. Res. 6, 601 (2018).

15. X. Hu, W. Zhang, Z. Yang, Y. Wang, W. Zhao, X. Li, H. Wang, C. Li, and D. Shen, "High average power, strictly all-fiber supercontinuum source with good beam quality," Opt. Lett. 36, 2659 (2011).

16. P. H. Pioger, V. Couderc, P. Leproux, and P. A. Champert, "High spectral power density supercontinuum generation in a nonlinear fiber amplifier," Opt. Express 15, 11358 (2007).

17. Q. Hao and H. Zeng, "Cascaded four-wave mixing in nonlinear Yb-doped fiber amplifiers," IEEE J. Sel. Top. Quantum Electron. 20, 345 (2014).

18. R. Song, J. Hou, S. P. Chen, W. Q. Yang, T. Liu, and Q. S. Lu, "Near-infrared supercontinuum generation in an all-normal dispersion MOPA configuration above one hundred watts," Laser Phys. Lett. 10, 015401 (2013).
19. R. Ma, Y. J. Rao, W. L. Zhang, X. Zeng, X. Dong, H. Wu, Z. N. Wang, and X. P. Zeng, "Backward supercontinuum generation excited by random lasing," IEEE J. Sel. Top. Quantum Electron. 24, 0901105 (2018).

20. R. Ma, W. L. Zhang, J. Y. Guo, and Y. J. Rao, "Decoherence of fiber supercontinuum light source for speckle-free imaging," Opt. Express 26, 26758 (2018).

21. L. Chen, R. Song, C. Lei, W. Yang, and J. Hou, "Random fiber laser directly generates visible to near-infrared supercontinuum," Opt. Express 27, 29781 (2019).

22. J. He, R. Song, W. Yang, and J. Hou, "High-efficiency ultra-compact nearinfrared supercontinuum generated in an ultrashort cavity configuration," Opt. Express 29, 19140 (2021).

23. H. Xu, M. Jiang, P. Zhou, G. Zhao, and X. Gu, "Elimination of self-modelocking pulses in high-power continuous-wave $\mathrm{Yb}$-doped fiber lasers with external feedback," Appl. Opt. 56, 9079 (2017).

24. W. Liu, P. Ma, P. Zhou, and Z. Jiang, "Optimization for the fiber laser source through its temporal and spectral characteristics," Proc. SPIE 10436, 1043600 (2017).

25. V. Bock, A. Liem, T. Schreiber, R. Eberhardt, and A. Tünnermann, "Explanation of stimulated Raman scattering in high power fiber systems," Proc. SPIE 10512, 105121F (2018).

26. A. E. Bednyakova, O. A. Gorbunov, M. O. Politko, S. I. Kablukov, S. V. Smirnov, D. V. Churkin, M. P. Fedoruk, and S. A. Babin, "Generation dynamics of the narrowband Yb-doped fiber laser," Opt. Express 21, 8177 (2013).

27. W. Liu, P. Ma, H. Lv, J. Xu, P. Zhou, and Z. Jiang, "General analysis of SRSlimited high-power fiber lasers and design strategy," Opt. Express 24, 26715 (2016).

28. J. Wang, J. Hu, L. Zhang, X. Gu, J. Chen, and Y. Feng, "A $100 \mathrm{~W}$ all-fiber linearly-polarized $\mathrm{Yb}$-doped single-mode fiber laser at $1120 \mathrm{~nm}$," Opt. Express 20, 28373 (2012).

29. L. Huang, P. Ma, R. Tao, C. Shi, X. Wang, and P. Zhou, "Experimental investigation of thermal effects and PCT on FBGs-based linearly polarized fiber laser performance," Opt. Express 23, 10506 (2015).

30. G. Agrawal, Nonlinear Fiber Optics (Academic Press, 2013).

31. M. Salhi, A. Hideur, T. Chartier, M. Brunel, G. Martel, C. Ozkul, and F. Sanchez, "Evidence of Brillouin scattering in an ytterbium-doped double-clad fiber laser," Opt. Lett. 27, 1294 (2002).

32. Y. Tang and J. Xu, "High-power pulsed thulium fiber oscillator modulated by stimulated Brillouin scattering," Appl. Phys. Lett. 104, 011103 (2014).

33. J. Xu, J. Wu, J. Ye, J. Song, B. Yao, H. Zhang, J. Leng, W. Zhang, P. Zhou, and Y. Rao, "Optical rogue wave in random fiber laser," Photon. Res. 8, 1 (2019). 